\title{
Erratum to: Charged residues next to transmembrane regions revisited: "Positive- inside rule" is complemented by the "negative inside depletion/outside enrichment rule"
}

\author{
James Alexander Baker ${ }^{1,2}$, Wing-Cheong Wong ${ }^{1}$, Birgit Eisenhaber ${ }^{1}$, Jim Warwicker ${ }^{2^{*}}$ and Frank Eisenhaber ${ }^{1,3^{*}}$
}

\section{Erratum}

Upon publication of the original article [1], the authors noticed that an error was introduced at proofing stage in the panel labels of Fig. 1. Instead of reading 'a; b; c; d', these read ' $a$; b; c; c'. This has now been updated in the original article and the correct version is included in this erratum. Inconsistencies in the use of brackets around the panel labels of the figure legends have also been corrected and each panel label is now displayed in brackets. We apologise for any inconvenience caused by these errors.

Please see below the correct Fig. 1:

\begin{abstract}
Author details
'Bioinformatics Institute, Agency for Science Technology and Research (A*STAR), 30 Biopolis Street \#07-01, Matrix, Singapore 138671, Singapore. ${ }^{2}$ School of Chemistry, Manchester Institute of Biotechnology, 131 Princess Street, Manchester M1 7DN, UK. ${ }^{3}$ School of Computer Engineering (SCE), Nanyang Technological University (NTU), 50 Nanyang Drive, Singapore 637553, Singapore.
\end{abstract}

Received: 25 July 2017 Accepted: 27 July 2017

Published online: 18 August 2017

\section{Reference}

1. $\quad$ Baker JA, Wong W;C, Eisenhaber B, Warwicker J and Eisenhaber F: Charged residues next to transmembrane regions revisited: "Positive-inside rule" is complemented by the "negative inside depletion/outside enrichment rule". BMC Biology 2017, 15:66. DOI: 10.1186/s12915-017-0404-4

\footnotetext{
* Correspondence: j.warwicker@manchester.ac.uk; franke@bii.a-star.edu.sg ${ }^{2}$ School of Chemistry, Manchester Institute of Biotechnology, 131 Princess Street, Manchester M1 7DN, UK

1 Bioinformatics Institute, Agency for Science Technology and Research

(A*STAR), 30 Biopolis Street \#07-01, Matrix, Singapore 138671, Singapore
} 
UniHuman

\section{a}

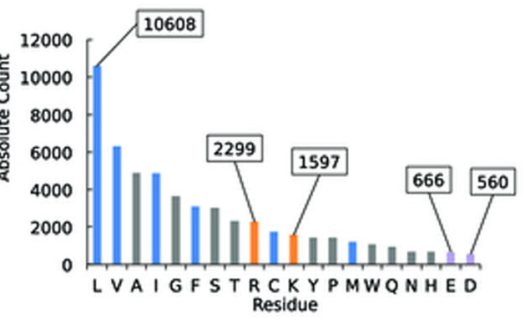

C

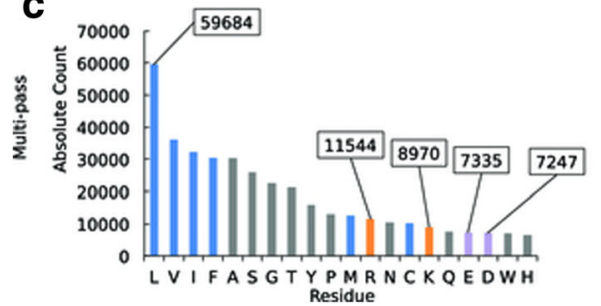

ExpAll

b

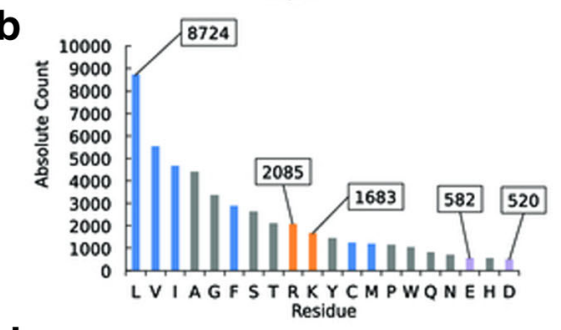

d

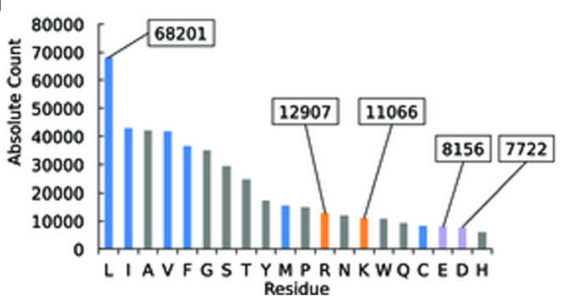

Fig. 1 Negatively charged amino acids are amongst the rarest residues in TMHs and \pm 5 flanking residues. Bar charts of the abundance of each amino acid type in the TMHs with flank lengths of the accompanying \pm 5 residues from the (a) UniHuman single-pass proteins, (b) ExpAll single-pass proteins, (c) UniHuman multi-pass proteins, and (d) ExpAll multi-pass proteins. Amino acid types on the horizontal axis are listed in descending count. The bars were coloured according to categorisations of hydrophobic, neutral and hydrophilic types according to the free energy of insertion biological scale [36]. Grey represents hydrophilic amino acids that were found to have a positive $\Delta$ Gapp, and blue represents hydrophobic residues with a negative $\Delta \mathrm{Gapp}$, purple denotes negative residues and positive residues are coloured in orange. The abundances of key residues are labelled 